\title{
Stability of equilibrium points in a predator-prey model with delayed argument
}

\author{
Maria Skvortsova \\ Sobolev Institute of Mathematics SB RAS \\ Novosibirsk, Russia \\ sm-18-nsu@yandex.ru
}

\begin{abstract}
We consider a system of differential equations with delayed argument, which describes the interaction between predator and prey populations. We consider questions of stability of equilibrium points and study asymptotic properties of solutions. We describe sets of initial vectorfunctions, for which solutions stabilize at infinity. We establish estimates of solutions characterizing the rate of stabilization. The results are obtained using modified Lyapunov-Krasovskii functionals.
\end{abstract}

Keywords - predator-prey model, delay differential equations, equilibrium points, asymptotic stability, estimates of solutions, attraction set, modified Lyapunov-Krasovskii functional

We consider a system of delay differential equations describing the interaction between predator and prey populations [1]:

$$
\begin{gathered}
x^{\prime}(t)=x(t)(r-a x(t)-b z(t)), \\
y^{\prime}(t)=k b x(t-\tau) z(t-\tau)-(D+\mu) y(t), \\
z^{\prime}(t)=D y(t)-v z(t),
\end{gathered}
$$

where $x(t)$ is the density of prey at time $t, y(t)$ is the density of immature predator at time $t, z(t)$ is the density of mature predator at time $t, r>0$ is the intrinsic growth rate of prey, $\mu>0$ is the death rate of immature predator and $v>0$

\author{
Timur Yskak \\ Sobolev Institute of Mathematics SB RAS \\ Novosibirsk, Russia \\ istima92@mail.ru
}

is the death rate of mature predator, constant $k>0$ denotes the coefficient in conversing prey into new immature predator, constant $\tau>0$ denotes the time delay due to gestation of mature predator, constant $D>0$ denotes the rate of immature predator becoming mature predator. The system originates from predator-prey model of Lotka-Volterra type.

We consider questions of stability of equilibrium points and study asymptotic properties of solutions to this system. We describe sets of initial vector-functions, for which solutions stabilize at infinity. We establish estimates of solutions characterizing the rate of stabilization. The results are obtained using modified Lyapunov-Krasovskii functionals [2].

\section{ACKNOWLEDGMENT}

The work is supported by Mathematical Center in Akademgorodok, the agreement with Ministry of Science and High Education of the Russian Federation number 07515-2019-1613.

\section{REFERENCES}

[1] W. Wang and L. Chen, "A predator-prey system with stage-structure for predator," Comput. Math. Appl., vol. 33(8), pp.83-91, 1997.

[2] G. V. Demidenko and I. I. Matveeva, "Asymptotic properties of solutions to delay differential equations," Vestn. Novosib. Gos. Univ., Ser. Mat. Mekh. Inform., vol. 5(3), pp. 20-28, 2005. 